\title{
The lysis of Trypanosoma brucei brucei by human serum
}

\author{
Stephen Tomlinson* and Jayne Raper ${ }^{1}$ \\ Departments of Pathology and 'Medical and Molecular Parasitology, NYU Medical Center, New York, NY 10016. \\ ${ }^{*}$ Corresponding author (e-mail: stephen.tomlinson@ccmail.med.nyu.edu).
}

Received 25 January 1996; accepted 4 April 1996.

\begin{abstract}
The natural immunity of humans to the cattle pathogen Trypanosoma brucei brucei, but not to the morphologically indistiguishable human pathogens $T$. brucei gambiense and $T$. brucei rhodesiense, is due to the selective killing of the parasite by normal human serum. The factor in human serum that mediates lysis of $T$. brucei brucei has long been attributed to a minor subclass of high density lipoprotein (HDL). Evidence indicates that the trypanolytic activity of isolated human HDL is due to peroxidase activity of an associated haptoglobin-related protein-hemoglobin complex. However, recent data suggest that the trypanolytic activity of HDL may be completely inhibited in whole human serum, and that trypanolytic activity of norman human serum is due to a second, less well-defined factor of high molecular weight. Current research aimed at understanding the mechamisms of cytotoxicity and the affected metabolic pathways may open new approaches for the development of specific drugs and vaccines against trypanosomiasis.
\end{abstract}

Keywords: Trypanosoma, high density lipoprotein, haptoglobin, serum lysis

\section{African trypanosomes and disease}

Trypanosomiasis is a major health and economic problem in Africa. The affected areas extend over more than 10 million $\mathrm{km}^{2}$ across the central region of the continent and threaten more than 50 million people and 25 million cattle. The main livestock pathogens are Trypanosoma congolense, Trypanosoma vivax, and Trypanosoma brucei brucei. Animals with trypanosomiasis have severe weakness, anemia, and cachexia. It has been estimated that as a result of this protozoan infection, Africa produces 70 times less animal protein per unit area than Europe. In areas of high tsetse fly density, even the animal stocks that are naturally more resistant to infection have to be treated to survive, and the susceptible cattle cannot be maintained even under chemoprophylaxis. Control of the tsetse vector is complicated and has proven impossible to achieve in practice, and vaccine development seems difficult because African trypanosomes can change their surface coat to escape attack by antibodies ${ }^{1,2}$. There is a wide variation in the natural susceptibility of cattle, sheep, goats, mice, and other animals to trypanosomiasis, and many animals show little parasitemia and survive the infection. The mechanism of this innate immunity appears complex and multigenic.

The human disease, known as sleeping sickness, is caused by T. brucei rhodesiense and T. brucei gambiense, which are transmitted by the bite of the tsetse fly (Glossina sp.). According to a World Development Report ${ }^{3}$, African sleeping sickness is the third most important contributor to the global burden of parasitic disease after malaria and schistosomiasis. More than 25,000 new cases are reported yearly, although the actual incidence is believed to be considerably higher. Unless treated, sleeping sickness is always fatal, and an acute form of the disease can lead to death in a few weeks. Chronic disease causes population displacements and social instability.
An intriguing phenomenon associated with natural immunity to these parasites is the complement-independent selective killing by normal human serum (NHS) of the cattle pathogen T. brucei brucei, but not of the morphologicaliy indistinguishable human pathogens T. brucei gambiense and T. brucei rhodesiense. Understanding the mechanism(s) by which T. brucei brucei is lysed by human serum, and the molecular basis for resistance of the human pathogens to human serum, may lead to the development of specific drugs and vaccines for both the human and animal disease.

\section{Lytic factors for T. brucei brucei in human serum}

There are at least two distinct trypanolytic factors in NHS. The first described, and best characterized, lytic factor is a minor subclass of high density lipoprotein (HDL); the second factor is not a lipoprotein and remains only poorly characterized. Although a subclass of HDL has long been regarded as the primary (if not the only) trypanolytic factor in NHS, recent evidence suggests that the nonlipoprotein factor is of more physiological relevance. Trypanolytic HDL will be refered to as trypanosome lytic factor 1 (TLF1), and the nonlipoprotein factor as TLF2.

Trypanosome lytic factor 1 . Since the discovery by Rifkin ${ }^{4}$ that an isolated fraction of human HDL is toxic for T. brucei brucei, many workers have corroborated her finding ${ }^{5-9}$. It is generally accepted that only a minor subclass of HDL kills trypanosomes, although there is some controversy regarding its precise nature $\mathrm{e}^{10,11}$. Different groups have reported the isolation of trypanolytic HDL subfractions that differ with regard to both size and density ${ }^{5,8,9}$. Nevertheless, the trypanolytic HDL particles (TLF1) isolated by Hajduk et al. ${ }^{5}$ remain the best characterized. These TLF1 particles are relatively large ( 15 to $21 \mathrm{~nm}, 490 \mathrm{kD}$ ) with a high density $(1.21$ to $1.24 \mathrm{~g} / \mathrm{ml})$. In addition to the major HDL-associated apolipoproteins (apo) AI and AII, the TLF1 particles contain 


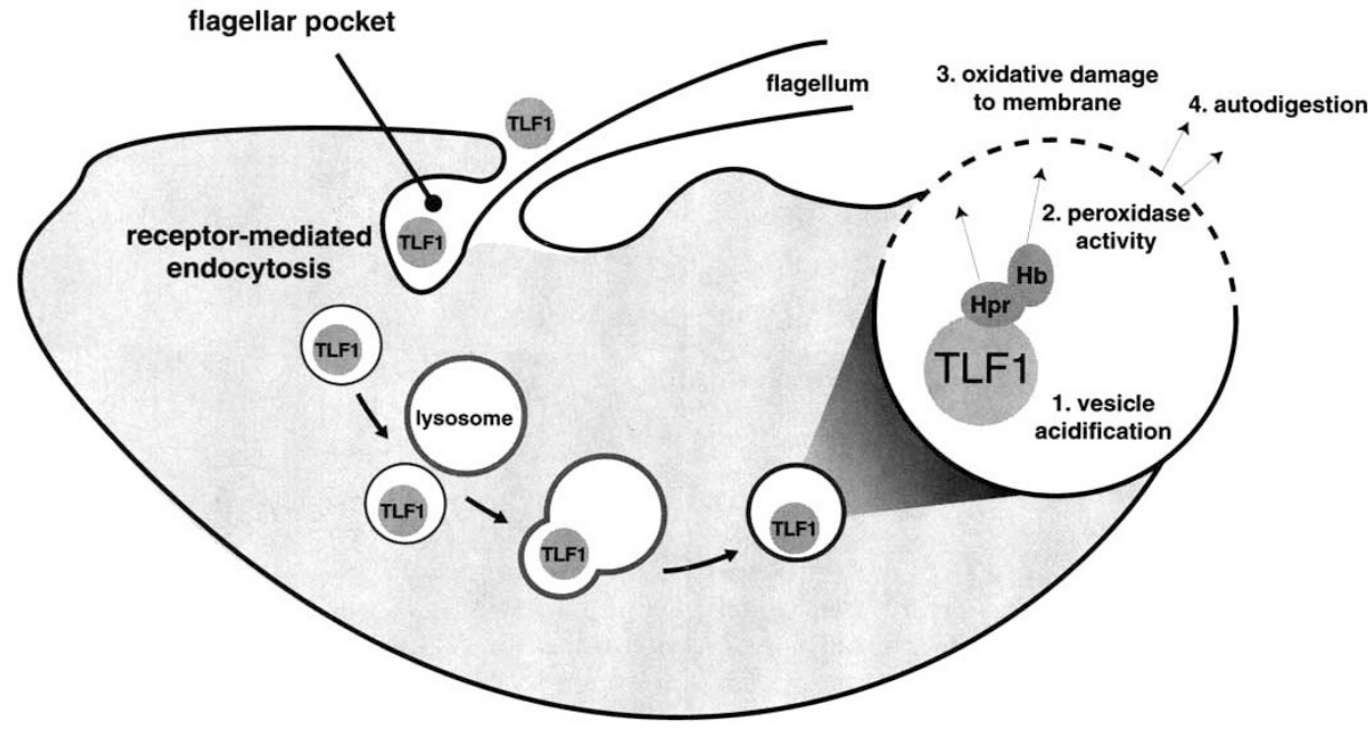

Figure 1. Schematic figure showing the mechanism of TLF1-mediated lysis proposed by Smith et al. ${ }^{12}$. See text for details.

haptoglobin-related protein (Hpr) and paraoxanase/arylesterase ${ }^{12}$. Smith et al. ${ }^{12}$ propose that like haptoglobin (Hp), TLF1-associated $\mathrm{Hpr}$ binds plasma hemoglobin $(\mathrm{Hb})$, and that the Hpr-Hb complex represents the toxic moiety of TLF1 (see below).

Trypanosome lytic factor 2 . About 10 years after the discovery of trypanolytic HDL, Barth ${ }^{13}$ described the isolation of a non-HDL trypanolytic factor (TLF2) from human serum. However, the presence of a nonlipoprotein serum lytic factor has been slow to gain acceptance, probably because the relationship between TLF2 and the much better characterized, and more active, TLF1 has remained obscure. Our recent data have corroborated some of the earlier findings of Barth, and shows that TLF2 is a high molecular weight complex $(>1000 \mathrm{kD})$ that does not float in density gradients ${ }^{13,14}$ and does not contain the HDL marker proteins apo AI or AII". Further confirmation that TLF1 and TLF2 are distinct lytic factors comes from the finding that $\mathrm{Hp}$ selectively inhibits only TLF $1^{14}$ (see below). The structure of TLF2 is unknown, and whether it contains $\mathrm{Hpr}$, or other components common to TLF1, remains to be established.

\section{Mechanisms of trypanosome lysis}

Our current understanding of the mechanism by which TLF1 lyses trypanosomes is due in large part to work done in the laboratory of Stephen Hajduk. Data described here indicate that the binding of TLF1 to trypanosomes is receptor-mediated, and that TLF1 exerts its toxic effect from an intracellular location following endocytosis. There is only a limited amount of data available concerning the mechanism of lysis by TLF2, but it appears likely that TLF2 is internalized and processed in a similar fashion to TLF1.

TLF1-mediated trypanolysis. Receptor-mediated endocytosis and uptake of macromolecules appear to occur from within the trypanosome flagellar pocket ${ }^{15}$, an invagination of the plasma membrane from which the flagellum emerges. The surface membrane of the flagellar pocket differs from the rest of the plasma membrane in that it contains relatively little variant surface glycoprotein (VSG), the major surface antigen that constitutes the protective coat of trypanosomes. Immunoelectron microscopic techniques have been used to show that gold-labeled transferrin ${ }^{16}$ and low density lipoprotein $(\mathrm{LDL})^{17}$ bind within the try- panosome flagellar pocket, from which they are endocytosed into coated vesicles and delivered to lysosomes. Similar studies using goldlabeled TLF1 have shown that at $4^{\circ} \mathrm{C}$ TLF1 binds to the flagellar pocket membrane, and at $17^{\circ} \mathrm{C}$ it is endocytosed into small coated vesicles. At $37^{\circ} \mathrm{C}$ these vesicles appear to fuse with larger vesicles presumed to be lysosomes, subsequent to which gold particles are observed in the cytoplasm $^{18}$. Trypanosome lysis appears to be temporally related to the release of TLF1 from the putative lysosomal compartment into the cytoplasm. Additional evidence for the important role of lysosomes in TLF1-mediated lysis is the observation that the lysosomotrophic agents chloroquine and $\mathrm{NH}_{4} \mathrm{Cl}$ prevent trypanolysis ${ }^{18,19}$. These agents prevent the acidification of the lysosome and therefore inhibit the activity of lysosomal proteases $^{20}$.

Cell surface receptors for $\mathrm{LDL}^{21,22}$ and transferrin ${ }^{23-26}$ have been identified and characterized in T. brucei brucei. These receptors are localized to the flagellar pocket and appear to be essentially invariant throughout the trypanosomatids ${ }^{15}$. Binding studies also indicate the presence of an HDL receptor ${ }^{18,27}$, and cysteine-rich acidic transmembrane protein-a potential HDL receptor-is located exclusively at the flagellar pocket ${ }^{28}$. The best evidence for the existence of a receptor for TLF1 lies in the observation that TLF1 binds to T. brucei brucei in a specific and saturable manner ${ }^{18}$. Data from binding experiments using purified TLF1 and purified nonlytic HDL indicate that trypanosomes contain a high-affinity TLF1-specific binding site and a low-affinity HDL binding site that can also bind TLF ${ }^{18}$. However, TLF1 receptor-related studies are complicated by the fact that complete separation of TLF1 from nonlytic HDL is difficult to achieve, and both of these particles appear to contain common ligands. Since trypanosomes contain between 22,000 (ref. 18) and 64,000 (ref. 28) binding sites for HDL, compared to an estimated 350 sites specific for TLF1 (ref. 18 ), the contamination of TLF1 with even very low concentrations of nonlytic HDL make it very difficult to distinguish between TLF1 binding to TLF1-specific receptors and binding to HDL receptors.

The ligand(s) involved in TLF1 uptake, which may differ from the toxic moeity of the particle, has not been identified. Reconstitution studies by Tytler et al. ${ }^{29}$ suggested that apo AI, a component of both bulk HDL and TLF1, could serve as a ligand. A trypanosome apo AI receptor would bind both TLF1 and nonlytic HDL, and may explain why nonlytic HDL partially competes with TLF1 for binding ${ }^{18}$. However, it is unlikely that an apo AI receptor plays a role in TLF1-mediated lysis since TLF1 represents $<1 \%$ of total serum HDL, yet total HDL preparations are fully trypanolytic; if an apo AI receptor were the only mode of entry for TLF1, its binding would be blocked by the large excess of nonlytic HDL. The finding that apo AI-deficient sera from patients with Tangier disease is trypanolytic ${ }^{9}$ also appears to exclude a role for apo AI in TLF1 lysis. A second candidate TLF1 ligand, which may 
bind to the TLF1-specific high-affinity binding site described by Hagar et al. ${ }^{18}$, is Hpr. Although direct evidence for its role as a ligand is lacking, such a function would offer a possible explanation for the ability of highly homologous $\mathrm{Hp}$ to inhibit TLF1-mediated lysis (see below). It is also possible that TLF1 is heterogeneous in nature, and can use multiple protein components as receptor ligands to ensure rapid access to the trypanosome interior.

Smith et al. ${ }^{12}$ recently identified Hpr as a unique component of TLF1, and have presented convincing evidence that TLF1mediated trypanosome lysis is the result of peroxidase activity associated with this protein. $\mathrm{Hpr}$ is over $90 \%$ identical to $\mathrm{Hp}^{30}$, an abundant plasma protein that binds free $\mathrm{Hb}$ with an extremely high affinity ${ }^{31}$. Smith et al. ${ }^{12}$ propose that TLF1-associated $\mathrm{Hpr}$ functions similarly to $\mathrm{Hp}$ in that it binds $\mathrm{Hb}$, and that $\mathrm{Hpr}-\mathrm{Hb}$ complexes exhibit peroxidase activity at low $\mathrm{pH}$. They have shown that purified TLF1 contains $\mathrm{Hb}$, possesses peroxidase activity at $\mathrm{pH} 4$ (albeit very low), and that TLF1-mediated trypanolysis can be inhibited by anti-Hp antibodies that cross-react with $\mathrm{Hpr}$. Furthermore, they have shown that the antioxidant enzyme catalase (which is not present in African trypanosomes) inhibits TLF1-mediated lysis, indicating that killing occurs via an oxidative mechanism. Their data support the following hypothesis for TLF1-mediated lysis (Fig. 1): Following internalization, TLF1 is targeted to acidic intracellular vesicles, which results in the expression of peroxidase activity by the TLF1 particle. The peroxidase activity causes oxidative damage to the vesicular membrane, which results in vesicle disruption, and the subsequent release of lysosomal enzymes into the cytoplasm leads to autodigestion of the parasite. It should be pointed out, however, that at physiologi$\mathrm{cal} \mathrm{pH}, \mathrm{Hp}$ is an antioxidant rather than an oxidant, and neutralizes the potentially toxic effects of $\mathrm{Hb}$. Only below $\mathrm{pH} 5.5$ does the association of $\mathrm{Hp}$ with $\mathrm{Hb}$ enhance peroxidase activity of $\mathrm{Hb}^{32}$. Recent data indicating a lysosomal $\mathrm{pH}$ of 6.1 in T. brucei rhodesiense ${ }^{33}$ appear to be in conflict with the hypothesis of TLF1-mediated trypanosome lysis proposed by Smith et al. However, further research is required to determine whether a similar lysosomal $\mathrm{pH}$ is found in other African trypanosomes. An intriguing possibility is that the lysosomal compartment of resistant trypanosomes is maintained at a $\mathrm{pH}$ higher than that of sensitive parasites.

TLF2-mediated tryponolysis. In contrast to TLF1, very little is known concerning the mechanism by which TLF2 kills T. brucei brucei. Partially purified TLF2 exhibits specific and saturable binding to T. brucei brucei, which suggests the presence of a TLF2 receptor (our unpublished data). In addition, TLF2 exhibits the same time- and temperature-dependent trypanolytic properties as TLF1. It should also be noted that earlier experiments relating to trypanosome lysis using whole human serum probably reflect the action of TLF2, since TLF1 appears to be inactive in complete NHS (see below). In common with TLF1, chloroquine and $\mathrm{NH}_{4} \mathrm{Cl}$ protect trypanosomes from lysis by TLF2. Together, these results indicate that the uptake of TLF2 is also the result of receptormediated endocytosis, and that killing requires the acidification of intracellular vesicles. It is not known whether TLF2 contains a ligand(s) distinct from TLF1. The characterization of a TLF2 receptor will require highly purified TLF2, especially as IgM and $\alpha-2$ macroglobulin are major contaminants of crude TLF2 preparations (our unpublished data), and both bind nonspecifically to trypanosomes ${ }^{34,35}$. The composition and strucure of TLF2 and the determination of the molecular basis of TLF2-mediated trypanolysis are central unresolved questions and present a challenge for future research.

\section{The role of $\mathrm{Hp}$ in serum-mediated trypanosome lysis}

The first indication that human serum contained an inhibitor of trypanolysis came from the observation that when TLF1 is sepa-

rated from the bulk of serum proteins, there is a considerable increase in its specific lytic activity ${ }^{5}$. Recent work has identified this inhibitor as the acute phase reactant $\mathrm{Hp}^{14,36}$, which is present in NHS at concentrations between 0.2 and $2 \mathrm{mg} / \mathrm{ml}$. Significantly, purified TLF1 is completely inhibited by concentrations of $\mathrm{Hp}$ below those found in $\mathrm{NHS}^{14,36}$. In contrast, the lytic activity of isolated TLF2 is unaffected by $\mathrm{Hp}^{14}$. These data indicate that TLF1 activity in NHS is completely inhibited by endogenous serum $\mathrm{Hp}$, and that the trypanolytic activity of NHS is most likely due solely to TLF2. Indeed, the addition of high concentrations of exogenous $\mathrm{Hp}$ to NHS does not affect its trypanolytic activity ${ }^{14}$, and there is no correlation between the trypanolytic activity of NHS and its endogenous $\mathrm{Hp}$ concentration ${ }^{37}$.

Further information regarding the effect of Hp on TLF1 and TLF2 activity in human serum came from studies of sera from patients suffering from intravascular hemolysis. These sera are depleted of $\mathrm{Hp}$ because the $\mathrm{Hb}$ released into the plasma binds $\mathrm{Hp}$, and the complexes are rapidly removed from the circulation by the liver ${ }^{31}$. Such $\mathrm{Hp}$-deficient sera are over tenfold more trypanolytic than $\mathrm{NHS}^{14,36}$. An unexpected, and so far unexplained finding, is that these Hp-depleted sera are also deficient in TLF2. Although TLF2 may be the main trypanolytic factor in NHS, the high TLF1dependent trypanolytic activity of $\mathrm{Hp}$-depleted sera may be of significance in areas where trypanosomiasis is endemic, since both trypanosomiasis and malaria cause intravascular hemolysis and thus Hp-depletion.

The inhibition of TLF1 by $\mathrm{Hp}$ is dose dependent and reversible ${ }^{36}$. It appears unlikely that inhibition is due to $\mathrm{Hb}$ displacement from TLF1-associated $\mathrm{Hpr}$, because both $\mathrm{Hp}$ and $\mathrm{Hp}-\mathrm{Hb}$ complexes are equally inhibitory. Also, the addition of excess $\mathrm{Hb}$ to NHS does not affect its trypanolytic activity ${ }^{14}$. It is also unlikely that $\mathrm{Hp}$ binds to TLF1 and blocks or displaces the ligand or toxic moiety, because when endogenous serum $\mathrm{Hp}$ is separated from TLF1 (by density or size), the lytic activity of TLF1 is recovered ${ }^{5,8,9}$. The remaining possibilities are that $\mathrm{Hp}$ binds to the TLF1 receptor and blocks uptake of TLF1, or that Hp reversibly inactivates the lytic component(s) of TLF1 either extra- or intracellularly. Unless there is a weak extracellular association between TLF1 and $\mathrm{Hp}$, it would appear more likely that inhibition occurs intracellularly, possibly following colocalization of $\mathrm{Hp}$ and TLF1 within intracellular vesicles. The mechanism of inhibition remains obscure.

\section{Mechanisms of trypanosome resistance to serum}

The mechanism(s) by which the human pathogens T. brucei rhodesiense and T. brucei gambiense resist lysis by human serum is not known. In fact, the only criteria often used to distinguish between T. brucei brucei and T. brucei rhodesiense is their resistance to nonimmune human serum, although this is not a stable characteristic for either parasite. The frequency of switching between serumresistant and serum-sensitive phenotypes has been calculated to be far greater than the mutation rate, and is therefore likely due to alternative gene expression ${ }^{38}$.

The bloodstream forms of African trypanosomes are able to

Table 1. Characteristics of isolated trypanolytic factors from human serum.

$$
\text { TLF1 }
$$
TLF2

- approximately $500 \mathrm{kD}$

- contains apo Al and All

- density = 1.17 to $1.25 \mathrm{~g} / \mathrm{ml}$

- inhibited by haptoglobin

- inhibited by chloroquine/ $\mathrm{NH}_{4} \mathrm{Cl}$

- $>1000 \mathrm{kD}$
- does not contain apo Al or All
- density $>1.250 \mathrm{~g} / \mathrm{ml}$
- not inhibited by haptoglobin
- inhibited by chloroquine $/ \mathrm{NH}_{4} \mathrm{Cl}$


evade the specific immune system of the host due to variation in their major surface antigen, VSG. The VSG genes are transcribed at one of about 20 different telomeric expression sites, each of which contains additional expression site-associated genes (ESAGs). There are at least eight ESAGs at each expression site, and most of them appear to encode surface proteins. A change in VSG expression is due either to the activation of a different expression site or to the switching of VSG genes on an active expression site (for review see ref. 2). It is highly unlikely that VSG is associated with serum susceptibility since both serum-sensitive and serum-resistant clonal populations expressing the same VSG have been reported ${ }^{38,39}$. However, some reports have indicated that switching between serum-resistant and serum-sensitive phenotypes was associated with a switch in VSG expression ${ }^{40,41}$, thus raising the possibility that ESAGs may be involved in the sensitivity of trypanosomes to human serum.

The data outlined in the preceeding sections indicate that parasites that are resistant to lysis by NHS are resistant to lysis by TLF2, since TLF 1 appears to be inactive in NHS. Some T. brucei rhodesiense strains are clearly resistant to both lytic factors, since it has been shown that a particular strain can be resistant to NHS and purified TLF $1^{8,9}$. However, at present it is not clear whether resistance to TLF1 and TLF2 is mediated via common effector pathways, or whether the mechanisms of resistance to each factor are independent.

Resistance to lysis by either TLF1 or TLF 2 could be due to an absent or altered receptor for the uptake of these lytic particles. With regard to resistance to TLF1, Lorenz et al. ${ }^{42}$ studied the binding of TLF1 to serum-resistant T. brucei rhodesiense. They showed that purified trypanolytic HDL3 $(\rho=1.12$ to $1.21 \mathrm{~g} / \mathrm{ml})$ binds equally to T. brucei brucei and T. brucei rhodesiense, and concluded that receptors for TLFl are present on both serum-sensitive and serum-resistant trypanosomes. However, trypanolytic $\mathrm{HDL}_{3}$ is likely to also contain nonlytic HDL, and the interpretation of such binding studies is difficult because of the large number of trypanosome HDL binding sites compared to the small number of putative TLF1-specific binding sites. TLF1 binding experiments with T. brucei gambiense have not been done, and whether TLF2 binds to T. brucei rhodesiense and T. brucei gambiense is also not known. Nevertheless, the incubation of T. brucei gambiense in NHS depletes it of trypanolytic activity ${ }^{43}$. Since the lytic activity in NHS for T. brucei brucei is attributed to TLF2, these findings imply that T. brucei gambiense binds TLF2.

Another possible mechanism of resistance to serum lytic factors is the inactivation of their activity by resistant parasites. TLF1 appears to mediate trypanosome lysis by oxidative damage. Thus, it is possible that resistance to TLF1 may be elicited by an increased antioxidative capacity of the parasite. For example, resistant parasites could express increased levels of trypanothione reductase, an enzyme localized in the cytosol of African trypanosomes and which constitutes part of their antioxidant system ${ }^{44}$.

Resistance to serum may be multigenic, and it is possible that more than one mechanism of resistance may exist. Indeed, it is now apparent that there is enormous diversity among the parasite populations causing infection in humans $\mathrm{s}^{45}$. Sexual recombination in $T$. brucei, which occurs in the insect vector ${ }^{10,46}$, is likely to contribute to this diversity. This method of genetic exchange thus provides a potential mechanism for the generation of new serumresistant trypanosome strains in the tsetse fly, following blood meals from multiple hosts infected with different parasite strains.

\section{Prospects}

The pathways leading to the lysis of trypanosomes by purified TLF1 are fairly well outlined. In contrast, little is known concerning the mechanism of action of TLF2, and its relationship to TLF1 remains obscure. A full understanding of the mechanisms of cytotoxicity and the affected metabolic pathways may open new approaches for development of specific drugs to treat trypanosomiasis. It will, of course, be of great interest to determine the molecular basis for the remarkable difference between T. brucei bruce $i$ and the human parasites $T$. brucei gambiense and T. brucei rhodesiense in their susceptibility to human serum. Do human pathogens lack TLF1 and/or TLF2 receptors or have mutant forms of the receptor, or are they not susceptible to the toxin(s)? If either of the latter two possibilities is true, and all species contain related receptors, ongoing research may open new perspectives for the development of a multisurface component vaccine, using trypanosome membrane receptor(s) for the lytic factors as immunogens. Receptors, presumably found in the flagellar pocket, are accessible to high molecular weight toxic moieties, and therefore should also be accessible to serum antibodies. Even if TLF receptors are absent from the human parasites, a vaccine for the immunization of cattle may be feasible. It will be interesting to see if transgenic animals expressing human $\mathrm{Hpr}$, or other identified proteins involved in the trypanolytic process, can resist infection by $T$. brucei brucei. If so, genetically engineered cattle resistant to infection may provide a viable approach in combating animal trypanosomiasis.

\section{Acknowledgments}

We are very grateful for the help provided by $V$. Nussenzweig in the preparation of this review. We also thank M.R. Rifkin, S.L. Hajduk, M.G.-S. Lee, and P. Lorenz for valuable discussions and the sharing of unpublished data. Work in this laboratory is supported by the UNDP/World Bank/WHO Special Programme for Research and Training in Tropical Diseases (TDR).

1. Borst, P. 1986. Discontinuous transcription and antigenic variation in try panosomes. Ann. Rev. Biochem. 55:701-732.

2. Pays, E., Vanhamme, L., and Berberof, M. 1994. Genetic controls for the expression of surface antigens in African trypanosomes. Ann. Rev. Microbiol. 48:25-52.

3. World Development Report. 1993. Investing in Health World Development Indication. The World Bank 329.

4. Rifkin, M.R. 1978. Identification of the trypanocidal factor in normal human serum: High density lipoprotein. Proc. Natl. Acad. Sci. USA 75:3450-3454.

5. Hajduk, S.L., Moore, D.R., Vasudevacharya, J., Siqueira, H., Torri, A.F., Tytler, E.M., and Esko, J.D.. 1989. Lysis of Trypanosoma brucei by a toxic subspecies of human high density lipoprotein. J. Biol. Chem. 264:5210-5217.

6. Seed, J.R. and Sechelski, J.B. 1989. Nature of the trypanocidal factor in human serum. J. Parasitol. 75:1003-1006.

7. Gillet, M.P.T. and Owen, J.S. 1991. Trypanosoma brucei brucei: differences in the trypanocidal activity of human plasma and its relationship to the level of high density lipoproteins. Trans. R. Soc. Trop. Med. Hyg. 85:612-616.

8. Lorenz, P., James, R.W., Owen, J.S., and Betschart, B. 1994. Heterogeneity in the properties of the trypanolytic factor in normal human serum. Mol. Biochem. Parasitol. 64:153-164.

9. Tomlinson, S., Jansen, A.-M., Koudinov, A., Ghiso, J.A., Choi-Miura, N., Rifkin M.R., Ohtaki, S., and Nussenzweig, V. 1995. High-density-lipoprotein-independent killing of Trypanosoma brucei by human serum. Mol. Biochem. Parasitol. 70:131-138.

10. Hajduk, S.L., Hagar, K.M., and Esko, J.D. 1994. Human high density lipoprotein killing of African trypanosomes. Ann. Rev. Microbiol. 48:139-162.

11. Lorenz, P., Betschart, B., and Owen, J.S. 1995. Trypanosoma brucei brucei and high density lipoproteins: old and new thoughts on the identity and mechanism of the trypanocidal factor in human serum. Parasitol. Today 11:348-352.

12. Smith, A.B., Esko, J.D., and Hajduk, S.L. 1995. Killing of trypanosomes by the human haptoglobin-related protein. Science 268:284-286.

13. Barth, P. 1989. A new method for the isolation of the trypanocidal factor from normal human serum. Acta Tropica 46:71-73.

14. Raper, J., Nussenzweig, V., and Tomlinson, S. 1996. The main lytic factor of Trypanosoma b. brucei in normal human serum is not high density lipoprotein. J. Exp. Med. 183:1023-1029.

15. Overath, P., Chaudhri, M., Steverding, D., and Ziegelbauer, K. 1994. Invariant surface proteins in bloodstream forms of Trypanosoma brucei. Parasitol. Today 10:53-58.

16. Steverding, D., Stierhof, Y.-D., Fuchs, H., Tauber, R., and Overath, P. 1995. Transferrin-binding protein complex is the receptor for transferrin uptake in Trypanosoma brucei. J. Cell Biol. 131:1173-1182.

17. Coppens, I., Opperdoes, F.R., Courtoy, P.J., and Baukhuin, P. 1987. Receptor mediated endocytosis in the bloodstream form of Trypanosoma brucei. Journal of Protozoology 34:465-473.

18. Hagar, K.M., Pierce, M.A., Moore, D.R., Tytler, E.M., Esko, J.D., and Hajduk, S.L. 1994. Endocytosis of a cytotoxic human high density lipoprotein results in 
disruption of acidic intracellular vesicles and subsequent killing of african trypanosomes. J. Cell Biol. 126:155-167.

19. Lorenz, P., Barth, P.E., Rudin, W., and Betschart, B. 1994. Importance of acidic intracellular compartments in the lysis of Trypanosoma brucei brucei by normal human serum. Trans. R. Soc. Trop. Med. Hyg. 88:487-488.

20. Seglen, P.O. 1983. Inhibitors of lysosomal function. Meth. Enzymol. 96:737-764

21. Bastin, P., Coppens, I., Saint-Remy, J.-M., Baudhuin, P., Opperdoes, F.R., and Courtoy, P.J. 1994. Identification of a specific epitope on the extracellular domain of the LDL-receptor of Trypanosoma brucei brucei. Mol. Biochem. Parasitol. 63:193-202.

22. Coppens, I., Bastin, P., Courtoy, P.J., Baudhuin, P., and Opperdoes, F.R. 1991. A rapid method purifies a glycoprotein of $\mathrm{Mr} 145000$ as the LDL receptor of Trypanosoma brucei brucei. Biochem. Biophys. Res. Comm. 178:185-191.

23. Salmon, D., Geuskens, M., Hanocq, F., Nolan, D., Ruben, L., and Pays, E. 1994 A novel heterodimeric transferrin receptor encoded by a pair of VSG expression site-associated genes in T. brucei. Cell 78:75-86.

24. Ligtenberg, M.J.L., Bitter, W., Kieft, R., Steverding, D., Janssen, H., Calafat, J., and Borst, P. 1994. Reconstitution of a surface transferrin binding complex in insect form Trypanosoma brucei. EMBO J. 13:2565-2573.

25. Steverding, D., Stierhof, Y.-D., Chaudhri, M., Ligtenberg, M., Schell, D., BeckSickinger, A.G., and Overath, P. 1994. ESAG 6 and 7 products of Trypanosoma brucei form a transferrin binding protein complex. European Journal of Cell Biology 64:78-87.

26. Grab D.J. Shaw, M.K. Wells, C.W. Verjee, V. Russo, D.C.W. Webster, P Naessens, J., and Fish, W.R. 1993. The transferrin receptor in African trypanosomes: identification, partial characterization and subcellular localization. European Journal of Cell Biology 62:114-126.

27. Gillet, M.PT and Owen, J.S. 1992. Characteristics of the binding of human and bovine high-density lipoproteins by bloodstream forms of the African trypanosome, Trypanosoma brucei brucei. Biochim. Biophys. Acta 1123:239-248.

28. Lee, G.-S.M., Bihain, B.E., Russell, D.G., Deckelbaum, R.J., and van der Ploeg, L.H.T. 1990. Characterization of a cDNA encoding a cysteine-rich cell surface protein located in the flageller pocket of the protozoan Trypanosoma brucei. Molecular and Cellular Biology 10:4506-4517.

29. Tytier, E.M., Moore, D.R., Pierce, M.A., Hagar, K.M., Esko, J.D., and Hajduk, S.L. 1994. Reconstitution of the trypanosome lytic factor. The role of lipids and apolipoproteins in the cytotoxicity of a subspecies of human high density lipoproteins. Mol Biochem. Parasitol. In press.

30. Maeda, N. 1985. Nucleotide sequence of the haptoglobin and haptoglobinrelated gene pair. J. Biol. Chem. 260:6698-6709.

31. Pintera, J. 1971. The biochemical, genetic, and clinicopathological aspects of haptoglobin. Williams \& Wilkens, Baltimore.
32. Kawamura, K, Kagiyama, S., Ogawa, A., and Yanase, T. 1972. Kinetics of peroxidase activity, absorption spectra and oxygen affinity of human hemoglobinhaptoglobin 1-1 complexes. Biochim. Biophys. Acta 285:22-27.

33. Brickman, M.J., Cook, J.M., and Balber, A.E. 1995. Low temperature reversibly inhibits transport from tubular endosomes to a perinuclear, acidic compartment in African trypanosomes. J. Cell Sci. 108:3611-3621.

34. Dwyer, D.M. 1976. Immunologic and fine structure evidence of avidly bound host serum proteins in the surface coat of a bloodstream trypanosome. Proc. Natl. Acad. Sci. USA 73:1222-1226.

35. Verducci, G. Perito, S., Rossi, R., Mannarino, E., Bistoni, F., and Marconi, P. 1989. Identification of a trypanocidal factor against Trypanosoma equiperdum in normal human serum. Parasitol. 98:401-407.

36. Smith, A.B. and Hajduk, S.L. 1995. Identification of haptoglobin as a natura inhibitor of trypanocidal activity in human serum. Proc. Natl. Acad. Sci. USA 92:10262-10266.

37. Raper, J., Nussenzweig, V., and Tomlinson, S, 1996. Lack of correlation between haptoglobin concentration and trypanolytic activity of normal human serum. Mol. Biochem. Parasitol. 76:337-338.

38. Rifkin, M.R., DeGreef, C., Jiwa, A., Landsberger, F.R., and Shapiro, S.Z. 1994 Human serum-sensitive Trypanosoma brucei rhodesiense: a comparison with serologically indentical human serum-resistant clones. Mol. Biochem. Parasitol. 66:211-220.

39. Hagar, K.M. and Hajduk, S.L. 1995. Cloning and characterization of human sera reistant and sensitive strains of $T$. b. hodesiense. Woods Hole, MA. abstract 79.

40. Ortiz, J.C., Sechelski, J.B., and Seed, J.R. 1994. Characterization of human serum-resistant and serum sensitive clones from a single Trypanosoma brucei gambiense parental clone. J. Parasitol. 80:550-557.

41. De Greef, C. and Hamers, R. 1994. The serum reistance-associated (SRA) gene of Trypanosoma brucei rhodesiense encodes a variant surface glycoprotein-like protein. Mol. Biochem. Parasitol. 68:277-284.

42. Lorenz, P., Owen, J.S., and Hassall, D. 1995. Human serum resistant Trypanosoma brucei modesiense accumulates similar amounts of fluorescently-labelled trypanolytic $\mathrm{HDL}_{3}$ particles as human serum sensitive $T$. b. brucei. Mol. Biochem. Parasitol. 74:113-118.

43. Ortiz-Ordonez, J.C. and Seed, J.R. 1995. The removal of trypanolytic activity from human serum by Trypanosoma brucei gambiense and its subsequent recovery in trypanosome lysates. J. Parasitol. 81:555-558.

44. Fairlamb, A.H. and Cerami, A. 1992. Metabolism and function of trypanothione in the kinetoplastida. Ann. Rev. Microbiol. 46:695-729.

45. Molyneux, D.H. Pentreath, $V_{1}$, and Doua, F 1996. pp. 1171-1196 in Manson's tropical diseases, vol. 20. Cook, G.C. (ed.). W.B. Saunders, London.

46. Schweizer, J., Tait, A., and Jenni, L. 1988. The timing and frequency of hybrid formation in African trypanosomes during cyclical transmission. Parasitol. Res. 75:98-101.

\section{BioPro Designer}

Now you can handle

Process Simulation, Scheduling and Economic Evaluation using a single tool, specifically designed for the needs of the Biochemical, Pharmaceutical, Food and Specialty Chemical Industries.

\section{BioPro Designer is available for the MS Windows platform}

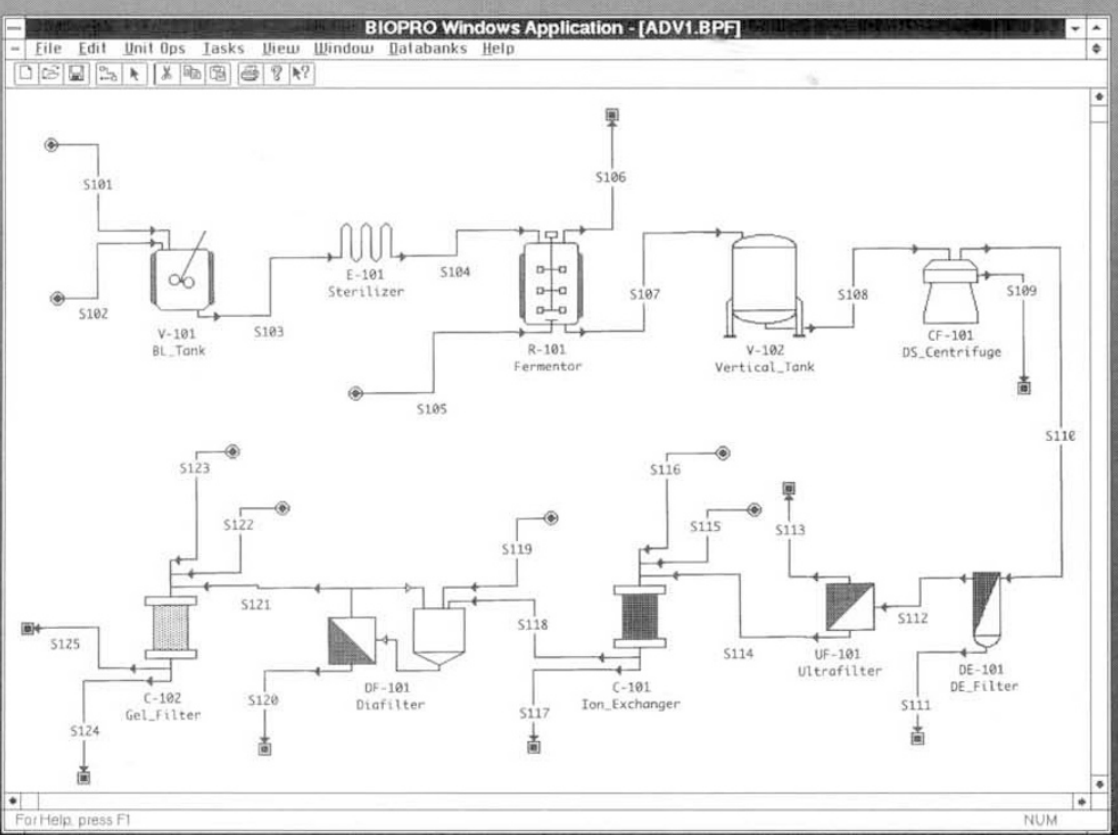

"A tremendous first step towards a successful design." - Harry Tbanos, VP of Process Technology, Sverdrup Corp.
BioPro Designer is used by over 100 companies and 150 universities worldwide

FEATURES

- Interactive graphical interface

- Models for over 60 unit operations

- Extensive component database

- Material and energy balances

- Estimation of equipment size and purchase cost

- Detailed simulation reports

- Detailed economic evaluation reports

- Scheduling of batch operations

- Hypertext on-line help

- Export PFDs to drawing packages

- Export reports to spreadsheets/word processors

- Create graphical objects (NEW)

- OLE-2.0 support (NEW)

\section{BENEFITS}

- Speed-up development and reduce cost

- Improve team communication

- Minimize environmental impact

For more information, please contact:

INTELUIGEN, INC.

2326 Morse Avenue • Scotch Plains • NJ 07076 Tel: (201) 622-1212 or (908) 654-0088

Fax: (908) 654-3866

E-mail: sales@intèlligen.com

WWw: http://www.intelligen.com

READER INQUIRY NO. 291 